Session 2509

\title{
Hydraulic Lung Analogue
}

\author{
Narciso F. Macia, Amy Gowder \\ Arizona State University
}

\begin{abstract}
This paper describes an easy-to-build, hydraulic analogue of the lungs, which has similar mechanical characteristics as an infant lung. It consist of two, clear, U-tubes filled with water (similar to two water-manometers) and connected through two capillary bundles into a T. Since the analogue is passive (it represents an unconscious subject), it is connected to a ventilator to demonstrate its characteristics. As the air enters through the T, it splits into two pathways (representing the first bifurcation present the trachea), and then passes through two capillary bundles, simulating airway resistance. The other sides of the capillary bundles are connected to the U-tubes, filled with water halfway. Since it is the tendency of the U-tube manometer is to maintain the two water columns at the same level, it provides a recoil effect that simulates lung compliance. The airway resistance can be changed by changing the size and/or number of the small tubes that make the capillary bundle. Lung compliance can be changed by selecting the size of the U-tube, or by placing a solid insert into one or both of U-tubes.

This device provides an adequate model for an infant lung provided that the frequency of excitation is not too high. This lung analog is an excellent vehicle for demonstrating the air movement in the respiratory system, since by using clear PVC piping, the water level change is equivalent to the tidal volume. The respiratory system provides excellent example of a dynamic bio-system and a vehicle to model dynamic systems.

Introduction

The respiratory system is an excellent vehicle for conveying the characteristics of a dynamic system. It provides enough complexity and analogous relationships to make the effort challenging, and also allows the student to work on a system that they are familiar with since they carry it everywhere they go. This exercise offers students the capacity to learn modeling techniques, testing methods, and critical understanding of the respiratory systems.

There are several approaches for modeling the respiratory system. These range from simply a tube connected to a balloon, to a system involving bellows ${ }^{1}$. There is also a commercially available device that allows the selection of compliance and resistance in each of its lobes ${ }^{2}$.

This paper presents another realization of the respiratory system, by utilizing two U-tube manometer tubes, to produce the recoil behavior present in the alveoli and the abdomen/chest wall. It consist of two, clear, U-tubes filled with water (similar to two water-manometers) and
\end{abstract}


connected through two capillary bundles into a T representing the trachea, as shown in Figure 1. Since the analogue represent an unconscious subject, it is normally connected to a ventilator to demonstrate its functioning characteristics.

As the air enters into the analogue, it splits into two pathways (representing the first bifurcation present the trachea), and then passes through two capillary bundles, simulating airway resistance. Notice that this allows modeling of asymmetrical resistances. The other sides of the capillary bundles are connected to the U-tubes, filled with water halfway. Since it is the tendency of the U-tube manometer is to maintain the two water columns at the same level, it provides a recoil effect that simulates respiratory system compliance.

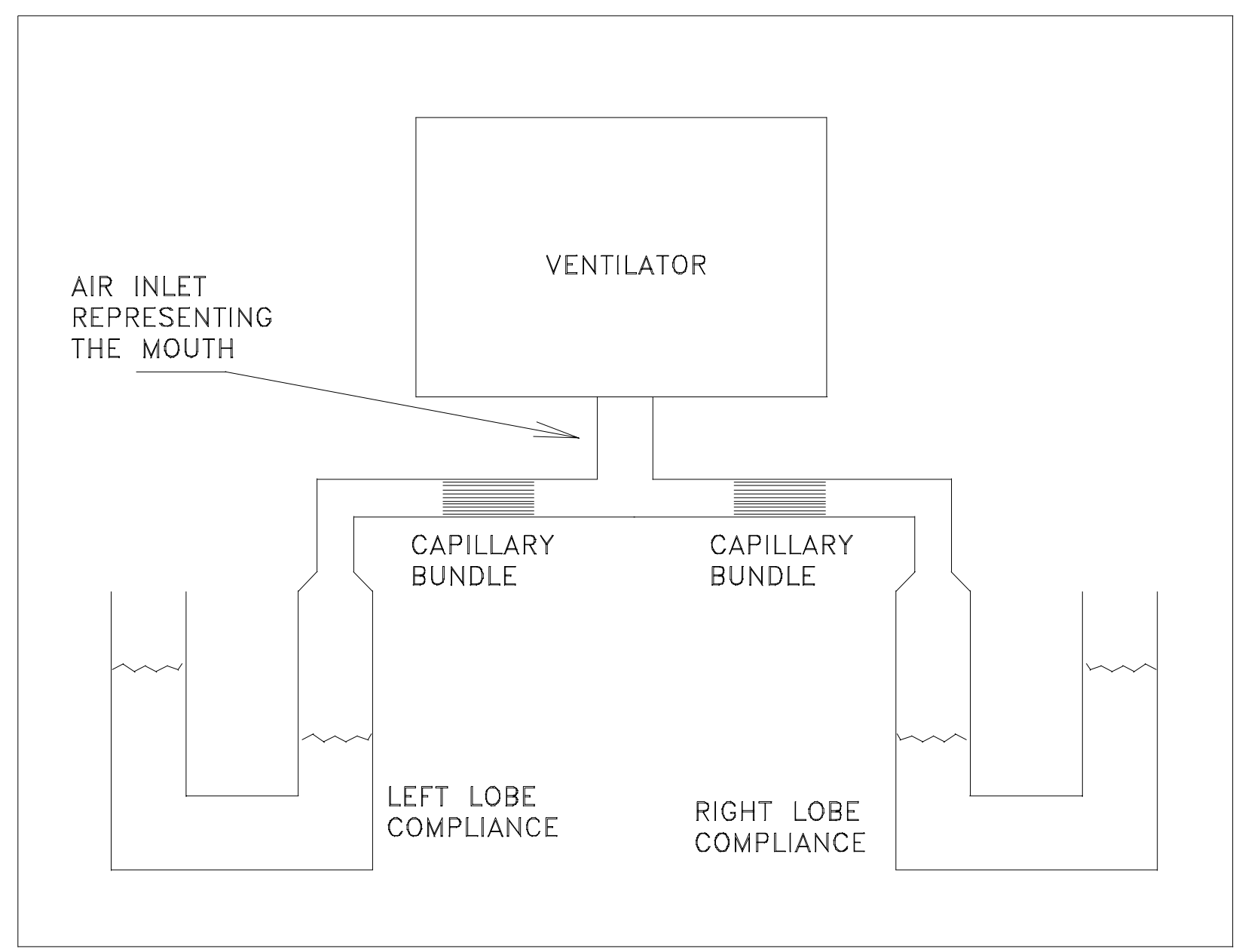

Figure 1: Schematic of U-tube lung analogue.

This analogue offers much value from an educational perspective. It is an excellent vehicle for teaching modeling and simulation of dynamic systems. It also lends itself to something that can be built with inexpensive components, allowing the students to excuse their building ability. Once the system is built and operational, it can provide much insight regarding the dynamics of the respiratory system. It effectively communicates the consequences of any asymmetry. If instrumented and modeled properly, it allows comparison of a real system and its corresponding model. Finally, the resulting analogue, like other devices, provides an excellent training tool for 
respiratory therapists. It is much more desirable for the respiratory therapist to familiarize himself with a new ventilator, first with the lung analogue and subsequently with a real patient. The compliance of each lobe is given by:

$$
C_{\text {lobe }}=\frac{\frac{\pi}{4} D^{2}}{2 \rho g}
$$

where $\mathrm{D}$ is the inside diameter of tube, $\rho$ is the density of water and $g$ the acceleration of gravity. This analogue also allows the simulation of a system with asymmetrical lung compliances. This can be done by introducing a cylindrical insert into one of the tubes, as shown in Figure 2 . The resulting compliance for this configuration is:

$$
C_{\text {lobe }}=\frac{\frac{\pi}{4} D^{2}}{\left\{1+\frac{1}{1-\left(\frac{d}{D}\right)^{2}}\right\} \rho g}
$$

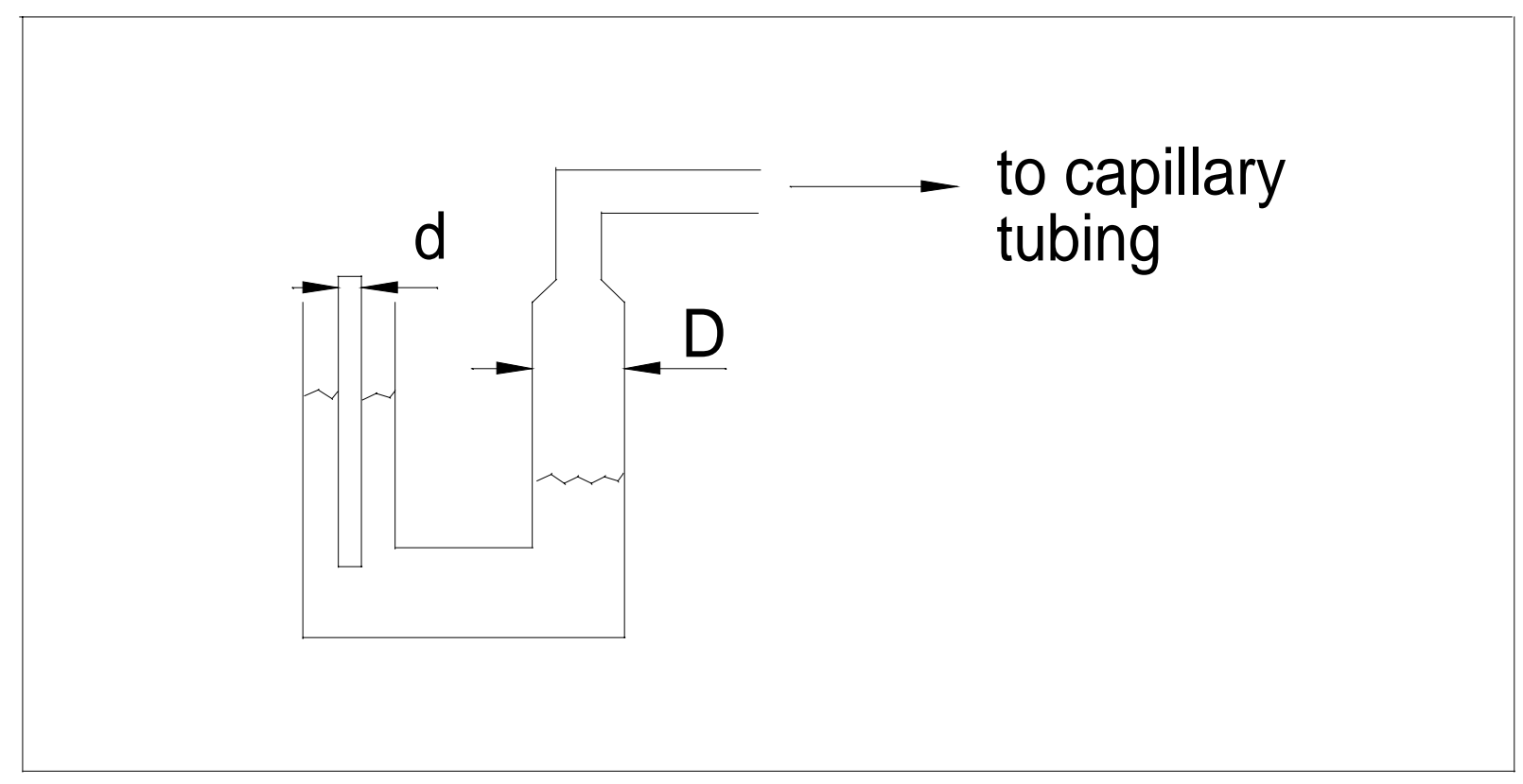

Figure 2: Method for changing capacitance.

Implementation of Resistances

It is desirable to obtain fluid resistances that exhibit a linear relationship between pressure drop and flow. One implementation that offers adequate linearity consists of gathering a bundle of small diameter tubes inside a plastic insert. Short capillary tubing used in blood analysis 
provides an inexpensive and effective method of obtaining these characteristics. Once the units are built, they are flow tested using a wet test meter. The pressure vs. flow data is plotted to insure adequate linearity in the range of use. The slope of the linear portion corresponds to resistance.

Mathematical description of the Model

The differential equation for the U-tube lung analog shown in Figure 1, assuming symmetry is:

$$
\frac{V}{C_{\text {total }}}+R_{\text {total }} \dot{V}+I_{\text {total }} \ddot{V}=P
$$

where $\mathrm{C}_{\text {total }}=2 \mathrm{C}_{\text {lobe }}, \mathrm{R}_{\text {total }}=\mathrm{R}_{\text {lobe }} / 2, \mathrm{I}_{\text {total }}=\mathrm{I}_{\text {lobe }} / 2$. Notice that the lobe parameters represent the characteristics of each side of the U-tube.

One way to describe the respiratory system is based on its behavior for different frequencies of excitation. Consider applying a sinusoidal pressure to the mouth of an unconscious subject. If the frequency of excitation is very low, the respiratory system acts primarily as a compliant chamber. In other words, neither the resistance nor the inertance affects the resulting volume into the lungs. For higher frequencies, the effect of resistance begins to surface. At the first break frequency, (1/RC), half the pressure goes to overcome resistance and the balance to overcome compliance. For even higher frequencies, the effect of resistance begins to dominate and afterward the effect of inertance becomes significant. For even higher frequency, inertance dominates.

Dynamic Characteristics

To compare the effectiveness of the U-tube analogue is describing an infant lung, we compare a model that describes the infant lung with the model for an equivalent U-tube analogue. For an infant, we assume the following parameters:

$$
\begin{aligned}
& C_{\text {inf }=} 2.5 \frac{\mathrm{cm}^{3}}{\mathrm{cmH}_{2} \mathrm{O}} \\
& R_{\text {inf }=} 0.040 \frac{\mathrm{cmH}_{2} \mathrm{O}}{\mathrm{cm}^{3} / \mathrm{s}} \\
& I_{\text {inf }=} 1.4 \times 10^{-4} \frac{\mathrm{cmH}_{2} \mathrm{O}}{\mathrm{cm}^{3} / \mathrm{s}^{2}}
\end{aligned}
$$

We can replicate the first two parameters in the U-tube analogue by selecting the U-tube diameter, D, and the proper capillary bundle. This represents a U-tube in which each lobe has half the compliance or

$$
C_{\text {lobe }}=1.25 \frac{\mathrm{cm}^{3}}{\mathrm{cmH}_{2} \mathrm{O}}
$$

This is obtained by using U-tubes with 0.703 " in diameter. However, this arrangement produces an inertance for each lobe of

$$
I_{\text {lobe }=} \frac{\rho l}{A}=1.1 \times 10^{-2} \frac{\mathrm{cmH}_{2} \mathrm{O}}{\mathrm{cm}^{3} / \mathrm{s}^{2}}
$$


Now, the total inertance is half the inertance of each lobe. As a result, the parameters corresponding to the complete U-tube analogue are:

$$
\begin{aligned}
& C_{U-t u b e=} 2.5 \frac{\mathrm{cm}^{3}}{\mathrm{cmH}_{2} \mathrm{O}} \\
& R_{U-\text { tube }=} 0.040 \frac{\mathrm{cmH}_{2} \mathrm{O}}{\mathrm{cm}^{3} / \mathrm{s}} \\
& I_{U-\text { tube }} 5.5 \times 10^{-3} \frac{\mathrm{cmH}_{2} \mathrm{O}}{\mathrm{cm}^{3} / \mathrm{s}^{2}}
\end{aligned}
$$

Notice that the resulting inertance is much higher than that of the infant. To compare the two models, we evaluate the effective compliance of each model by calculating the Bode plot of the transfer function:

$$
C_{e f f}=\frac{V}{P}=\frac{1}{1 / C+R s+I s^{2}}
$$

The results are shown in Figure 3.

Figure 3:
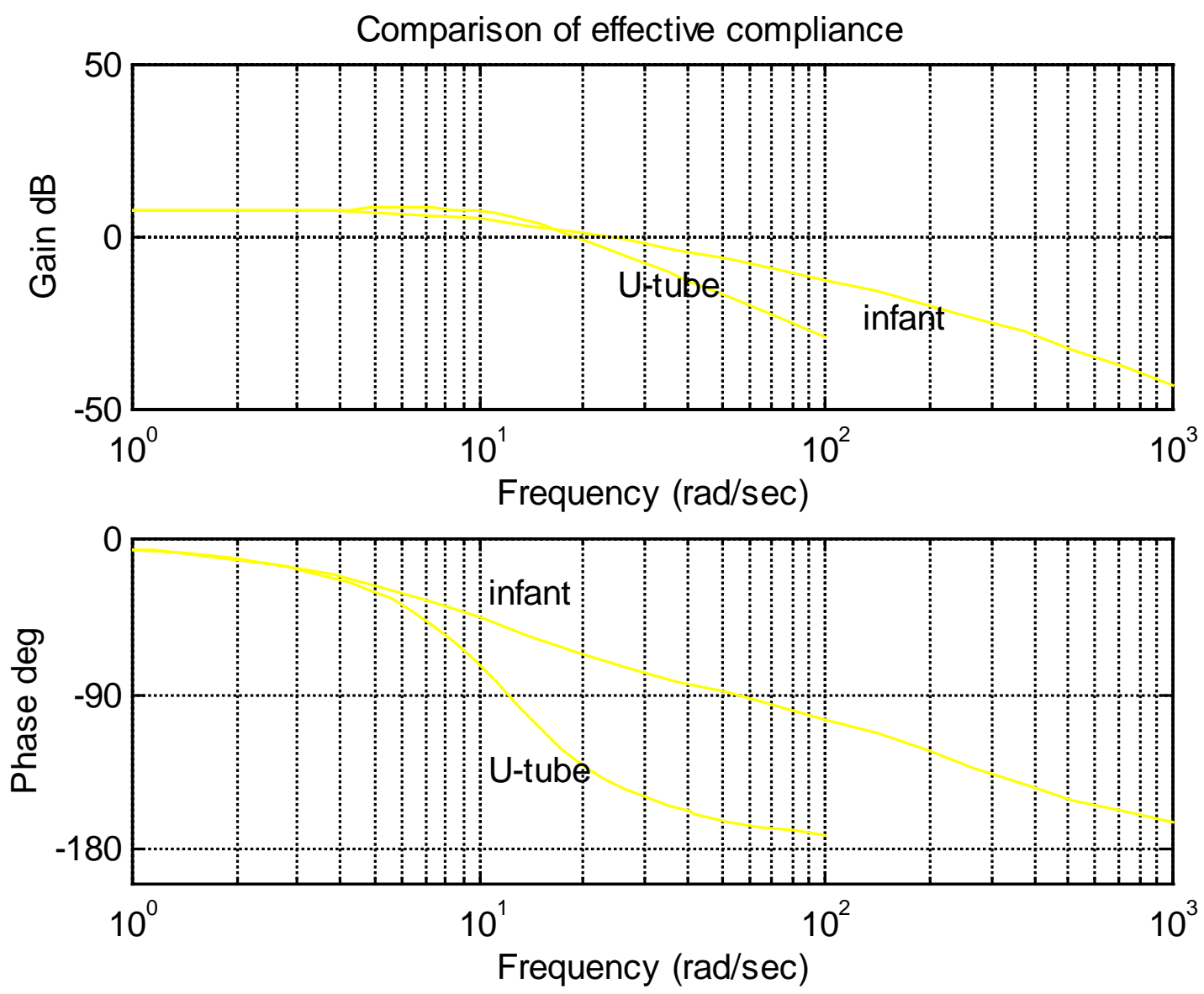
We also compare the reciprocal of effective resistance for the infant model and the U-tube, utilizing the following transfer function:

$$
\frac{1}{R_{e f f}}=\frac{\dot{V}}{P}=\frac{s}{1 / C+R s+I s^{2}}
$$

The results are shown in Figure 4.

Figure 4:
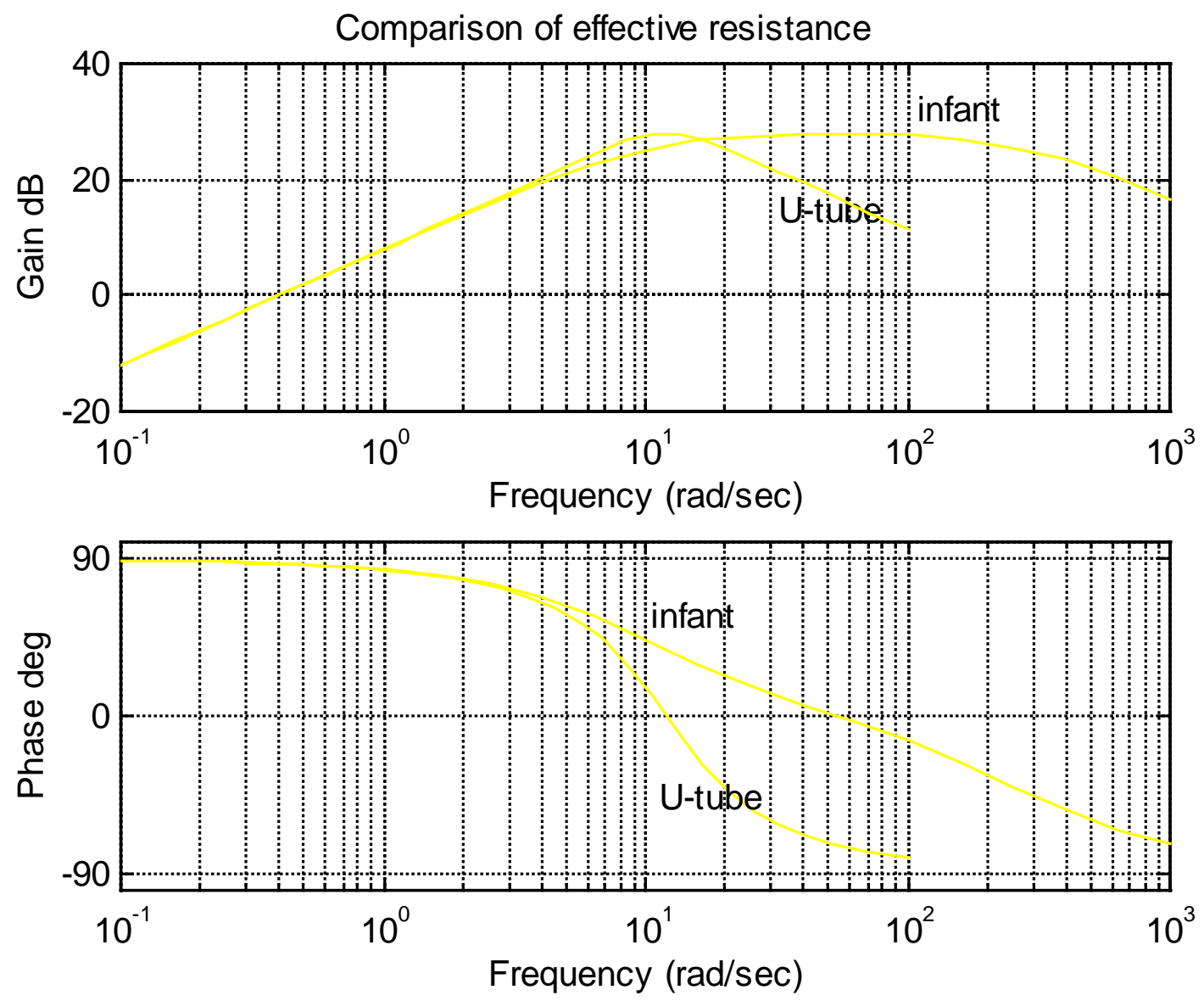

Notice that the infant and U-tube mode exhibit similar magnitudes up to about $20 \mathrm{rad} / \mathrm{s}(3 \mathrm{~Hz})$. Thus for frequencies below this upper limit, the U-tube does an adequate job in modeling the infant lung. The limitations of the model also provide an excellent way to challenge the students understanding of the respiratory system and modeling techniques. 


\section{Bibliography}

1. Verbraak, A.F.M., Beneken, J.E.W., Bogaard, J.M., Versprille, A., Computer-controlled mechanical lung model for application in pulmonary function studies, Med. 8 BID. Eng. \& Comput., 1995, 33, 776-783

2. Training Test Lung, Michigan Instruments, Inc., Grand Rapids, MI

\section{Narciso F. Macia}

Narciso F. Macia is an Associate Professor in the Electronics and Computer Engineering Technology Dept. Dr. Macia is a registered Mechanical Engineer in Arizona and is active in applied research grants. Dr. Macia received a B.S. degree in Mechanical Engineering from the University of Texas at Arlington in 1974, a M.S. degree in the Mechanical Engineering from the same department in 1976 and a Ph.D. in Electrical Engineering from Arizona State University in 1988.

Amy Gowder

Amy Gowder is an engineer at Anderson-Consulting in Arizona. Ms. Gouder was earning her B.S. degree in BioEngineering from Arizona State University at the time of this project. She graduated in 1998. 